OPEN ACCESS

Edited by:

Daniel Pinero,

National Autonomous University of

Mexico, Mexico

Reviewed by:

Ming Kang,

South China Botanical Garden - CAS,

China

Xue-jun Ge,

South China Botanical Garden - CAS,

China

Lev Jardón Barbolla,

National Autonomous University of

Mexico, Mexico

*Correspondence:

De-Zhu Li

dz@mail.kib.ac.cn

Lian-Ming Gao

gaolm@mail.kib.ac.cn

Specialty section: This article was submitted to Plant Genetics and Genomics,

a section of the journal

Frontiers in Plant Science

Received: 11 June 2016 Accepted: 05 August 2016 Published: 30 August 2016

Citation: Wambulwa MC Meegahakumbura MK, Kamunya S, Muchugi A, Möller M, Liu J, Xu J-C,

Ranjitkar S, Li D-Z and Gao L-M

(2016) Insights into the Genetic Relationships and Breeding Patterns of the African Tea Germplasm Based on nSSR Markers and cpDNA Sequences. Front. Plant Sci. 7:1244. doi: 10.3389/fpls.2016.01244

\section{Insights into the Genetic Relationships and Breeding Patterns of the African Tea Germplasm Based on nSSR Markers and cpDNA Sequences}

\author{
Moses C. Wambulwa 1, 2, 3, 4,5, Muditha K. Meegahakumbura, 2, 3, 6, Samson Kamunya ${ }^{5}$, \\ Alice Muchugi ${ }^{4}$, Michael Möller ${ }^{7}$, Jie Liu ${ }^{1}$, Jian-Chu Xu ${ }^{4,8}$, Sailesh Ranjitkar ${ }^{8}$, \\ De-Zhu $\mathrm{Li}^{1,2,3 *}$ and Lian-Ming Gao ${ }^{1 *}$ \\ ${ }^{1}$ Key Laboratory for Plant Diversity and Biogeography of East Asia, Kunming Institute of Botany, Chinese Academy of \\ Sciences, Kunming, China, ${ }^{2}$ Germplasm Bank of Wild Species in Southwest China, Kunming Institute of Botany, Chinese \\ Academy of Sciences, Kunming, China, ${ }^{3}$ College of Life Science, University of Chinese Academy of Sciences, Kunming, \\ China, ${ }^{4}$ Genetic Resources Unit, World Agroforestry Centre, Nairobi, Kenya, ${ }^{5}$ Tea Research Institute, Kenya Agricultural and \\ Livestock Research Organization, Kericho, Kenya, ${ }^{6}$ Genetic and Plant Breeding Division, Coconut Research Institute, \\ Lunuwila, Sri Lanka, ${ }^{7}$ Department of Science, Royal Botanic Garden Edinburgh, Edinburgh, UK, ${ }^{8}$ Centre for Mountain \\ Ecosystems, Kunming Institute of Botany, Chinese Academy of Sciences, Kunming, China
}

Africa is one of the key centers of global tea production. Understanding the genetic diversity and relationships of cultivars of African tea is important for future targeted breeding efforts for new crop cultivars, specialty tea processing, and to guide germplasm conservation efforts. Despite the economic importance of tea in Africa, no research work has been done so far on its genetic diversity at a continental scale. Twenty-three nSSRs and three plastid DNA regions were used to investigate the genetic diversity, relationships, and breeding patterns of tea accessions collected from eight countries of Africa. A total of 280 African tea accessions generated 297 alleles with a mean of 12.91 alleles per locus and a genetic diversity $\left(H_{S}\right)$ estimate of 0.652. A STRUCTURE analysis suggested two main genetic groups of African tea accessions which corresponded well with the two tea types Camellia sinensis var. sinensis and C. sinensis var. assamica, respectively, as well as an admixed "mosaic" group whose individuals were defined as hybrids of F2 and $\mathrm{BC}$ generation with a high proportion of $C$. sinensis var. assamica being maternal parents. Accessions known to be $C$. sinensis var. assamica further separated into two groups representing the two major tea breeding centers corresponding to southern Africa (Tea Research Foundation of Central Africa, TRFCA), and East Africa (Tea Research Foundation of Kenya, TRFK). Tea accessions were shared among countries. African tea has relatively lower genetic diversity. $C$. sinensis var. assamica is the main tea type under cultivation and contributes more in tea breeding improvements in Africa. International germplasm exchange and movement among countries within Africa was confirmed. The clustering into two main breeding centers, TRFCA, and TRFK, suggested that some traits of $C$. sinensis var. assamica and their associated genes possibly underwent selection 
during geographic differentiation or local breeding preferences. This study represents the first step toward effective utilization of differently inherited molecular markers for exploring the breeding status of African tea. The findings here will be important for planning the exploration, utilization, and conservation of tea germplasm for future breeding efforts in Africa.

Keywords: African tea germplasm, breeding improvement, Camellia sinensis, genetic diversity, nSSR markers, cpDNA regions

\section{INTRODUCTION}

Tea is one of the most popular beverages and has become a daily drink for billions of people across the world. The culture of drinking tea originated in China, and has been adopted in many parts of the world due to its numerous health benefits and attractive aroma (Yang et al., 2009; Bedran et al., 2015; Yin et al., 2015). The current global tea production stands at 5.35 million tons Food and Agricultural Organization of the United Nations (FAO, 2015a), with an estimated total sale of 10.84 billion U.S. dollars. Africa is one of the key centers for tea production, accounting for about $25 \%$ of the global black tea industry Food and Agricultural Organization of the United Nations (FAO, 2015a). The main tea producing countries in Africa include Kenya, Malawi, Uganda, Tanzania, Zimbabwe, South Africa, and Rwanda where tea contributes significantly to their respective economies. For instance, Kenya's tea exports earned the country around $\$ 1.33$ billion in 2013 Food and Agricultural Organization of the United Nations (FAO, 2014). It is projected that the continent's export volume will reach 743,384 metric tons by the year 2023 (FAOSTAT, 2015).

The first commercial tea plantation in Africa was established in 1878 in Malawi near Blantyre (Anonymous, 1962). However, it was not until 1956 that breeding work would begin in Malawi following the establishment of the Tea Research Foundation of Central Africa (TRFCA; Ellis and Nyirenda, 1995). Clone establishment started with field selections (FS) from established seedling populations in Swazi, Malawi. The seedling populations had been established earlier using a few founder cultivars from China and India (Eastern Produce Malawi Ltd. Personal interview. 4 March 2015). Clones selected in this way were denoted SFS (Swazi Field Selection) e.g., SFS 204 and SFS 150. These were used as parental resources, which eventually gave rise to the present tea germplasm of southern Africa through crossing and further selection. A few years later in 1961, breeding work commenced in Kenya under the oversight of the Tea Research Institute of East Africa which was in 1980 renamed Tea Research Foundation of Kenya (TRFK; Mondal, 2014). Historical records

\footnotetext{
Abbreviations: AFLP, Amplified fragment length polymorphism; BC1, Backcross to parent 1; BC2, Backcross to parent 2; cpDNA, Chloroplast DNA; CTAB, Cetyltrimethylammonium bromide; F1, First filial generation; F2, Second filial generation; FS, Field selections; H, Haplotype; MCMC, Markov chain Monte Carlo; nSSR, Nuclear simple sequence repeats; P1, Parent 1; P2, Parent 2; PCoA, Principal co-ordinates analysis; RAPD, Random amplified polymorphic DNA; SFS, Swazi field selection; TRFCA, Tea Research Foundation of Central Africa; TRFK, Tea Research Foundation of Kenya.
}

show that seeds of tea plants from India and Sri Lanka were used to establish the pioneer tea plantations in Kenya in early 1900s (Matheson and Bovill, 1950). Because these progenies had not been particularly selected for high yield and quality, the resultant seedling populations of mixed genotypes were phenotypically inferior, though diverse. Tea cultivar improvement started by mass selecting among the introduced seedlings to develop pioneer cultivars such as TRFK 6/8.

The tea plant, Camellia sinensis (L.) Kuntze, is a woody evergreen plant in the family Theaceae and is native to the region covering the northern part of Myanmar, and the provinces of Yunnan and Sichuan in China (Wight, 1959). Based on morphological features, Wight (1962) defined three tea types of cultivated tea, i.e., C. sinensis var. sinensis (China tea), C. sinensis var. assamica (Masters) Hung T. Chang (Assam tea), and its subspecies rank C. assamica subsp. lasiocalyx (Planchon ex Watt.) Wight (Cambod tea). These groups were later treated as one species with two varieties [C. sinensis var. sinensis and C. sinensis var. assamica (J. W. Mast.) Kitam., including C. assamica subsp. lasiocalyx as a synonym; Ming, 2000; Ming and Bartholomew, 2007]. Our recent study indicated that $C$. assamica subsp. lasiocalyx is a hybrid between $C$. sinensis var. sinensis and $C$. sinensis var. assamica, and not a distinct genetic entity (Wambulwa et al., 2016), and the cultivated tea plant includes three distinct lineages (Meegahakumbura et al., 2016). Efforts have been made previously to assess the genetic diversity, relationships, and population structure of the tea germplasm in Africa (Wachira et al., 1995; Paul et al., 1997; Yao et al., 2008; Wambulwa et al., 2016). However, none of these studies attempted to characterize the entire African tea germplasm using robust molecular markers. For instance, Wachira et al. (1995) and Paul et al. (1997) studied the genetic diversity using few accessions from Kenya based on random amplified polymorphic DNA (RAPD) and amplified fragment length polymorphism (AFLP), respectively. However, the low sample sizes and the use of dominant markers might have reduced the reliability of the results. Wambulwa et al. (2016) recently characterized the genetic diversity and relationships among 193 tea accessions from Kenya using SSR markers, which revealed that $C$. sinensis var. assamica is the most popular tea type in East Africa and harbored the lowest genetic diversity compared to other tea types. Although this study was a significant improvement compared to former studies, it is important to scale up the sampling area to sufficiently cover the African tea growing countries. However, the levels of genetic diversity, and germplasm exchanges of tea cultivars within African countries are still unknown. Given the common practice of germplasm exchanges within Africa, it is expected that 
C. sinensis var. assamica would still be the dominant tea type, and to have a low genetic diversity.

Nuclear microsatellite markers (nSSRs) are ubiquitously distributed in the genome, show a co-dominant inheritance, are highly polymorphic, frequently transferable across related species (Chase et al., 1996; Ellegren, 2004; Selkoe and Toone, 2006; Allan and Max, 2010; Fan et al., 2013), and have been widely used to assess the level of genetic variation in many plant species. Variation in chloroplast DNA (cpDNA) have also been used widely in population genetics and for parental identification (Kaundun and Matsumoto, 2011; Paule et al., 2012). CpDNA evolves slowly, has low mutation rates, no recombination, and is usually uniparentally inherited in angiosperms (Wolfe et al., 1987; Clegg and Zurawski, 1992; Mogensen, 1996). It is also known that cpDNA is maternally inherited in Theaceae (Yang et al., 2013). Thus, combined analysis of biparentally inherited nuclear SSR markers and maternally inherited cpDNA could provide an opportunity to investigate the genetic diversity and offer insights into the parental origin of unknown genetic pedigrees of the tea plant created in the process of genetic improvement of tea cultivars in Africa.

In the present study, we use nSSR genotyping, and cpDNA sequencing to investigate the genetic relatedness and breeding patterns of 280 tea accessions collected from eight countries across Africa (including Madagascar). The main objectives were to (1) determine the genetic diversity and structure of African tea at the continental scale, (2) establish the genetic relationships and movement of tea accessions within Africa, and (3) attempt to explore the breeding patterns and uncover differential selection between the TRFCA and TRFK breeding programmes in Africa. The results will provide baseline data that can have significant implications for the improvement of tea cultivars in Africa.

\section{MATERIALS AND METHODS}

\section{Sample Collection and DNA Extraction}

A total of 280 tea accessions were collected from eight African countries, including Kenya (183 samples), Rwanda (27), Tanzania (10), Malawi (20), South Africa (8), Cameroon (13), Nigeria (4), and Madagascar (15). Out of the total, 183 tea accessions sampled from Kenya represented the germplasm under cultivation in the entire East African region, i.e., Kenya, Uganda, Tanzania, Rwanda, and Burundi. The tea samples from Madagascar were collected from a deserted tea garden (located in Ambositra District) and morphologically identified as Camellia sinensis var. pubilimba Hung T. Chang, which occurs naturally in South China. The collection target was to cover the historic cultivation areas of southern and eastern Africa as well as the new entrants in western Africa. Details of all accessions are provided in Table S1. Healthy young leaves were sampled from randomly selected plants and immediately stored in silica-gel until DNA extraction. Total DNA was extracted following the cetyltrimethylammonium bromide (CTAB) method as described by Doyle and Doyle (1990).

\section{nSSR Genotyping}

Genotyping of the 280 tea accessions was carried out with a total of 23 highly polymorphic nSSRs employed in our previous study (Wambulwa et al., 2016). Visualization and sizing of the SSR fragments was performed using GENEMARKER v.4.0 (SoftGenetics LLC, State College, PA, USA). The genotyping procedure (amplification and fragment size determination) for all samples followed our previous study (Wambulwa et al., 2016). The nSSR data has been deposited at http://dx.doi.org/10.5061/dryad.3g02j in the Dryad Digital Repository.

\section{cpDNA Sequencing}

Three fast-evolving plastid intergenic spacer regions ( $n d h F-$ rpl32, trnSGG-trnSr and trnSf1-trnGGG) were sequenced for 84 accessions selected based on results of the nSSR data (Figure S1, Table S1, highlighted in yellow). The 84 accessions represented the various genetic groups in the nSSR NJ tree and origins from the different countries. We also included some known hybrids together with their parents in order to untangle the genetic pedigrees of some specific accessions. Primer sequences for the three regions are provided in Table S2. PCR amplification was carried out on a GeneAmp PCR System 9700 thermal cycler (PerkinElmer, Foster City, CA, USA). The $20 \mu \mathrm{L}$ PCR mix contained $2 \mu \mathrm{L}$ of $10 \times$ PCR buffer, $1.6 \mu \mathrm{L}$ of $25 \mathrm{mM} \mathrm{MgCl}_{2}$, $0.4 \mu \mathrm{L}$ of $10 \mathrm{Mm}$ dNTPs, $0.4 \mu \mathrm{L}$ of each primer (forward and reverse), $0.15 \mu \mathrm{L}$ of Taq polymerase (5 units $/ \mu \mathrm{L}$ ), $1 \mu \mathrm{L}$ of $40-$ $50 \mathrm{ng} / \mu \mathrm{L}$ of template DNA and $14.05 \mu \mathrm{L}$ sterile double-distilled water. The PCR conditions included an initial denaturation at $94^{\circ} \mathrm{C}$ for $3 \mathrm{~min}$, followed by 35 cycles of $30 \mathrm{~s}$ at $94^{\circ} \mathrm{C}$ for template denaturation, $30 \mathrm{~s}$ at $50^{\circ} \mathrm{C}$ for primer annealing, $50 \mathrm{~s}$ at $72^{\circ} \mathrm{C}$ for extension, and followed with an extension step of $10 \mathrm{~min}$ at $72^{\circ} \mathrm{C}$. PCR products were purified using ExoSAP-IT (GE Healthcare Life Sciences, USA). Purified PCR products were sequenced in both directions with the same primers used for PCR and then analyzed on an ABI 3730xl DNA Sequencer (Applied Biosystems, Forster city, USA). All the cpDNA sequences were deposited in GenBank and the accession numbers are provided in Table S1.

\section{Data Analysis nSSR Genotyping}

The automated genotyping results were re-checked manually. Genotyping errors such as the presence of null alleles, large allele drop-outs, and stuttering were examined by MICROCHEKER v2.2.3 (Van Oosterhout et al., 2004). The genetic indices, observed heterozygosity $\left(H_{O}\right)$, genetic diversity $\left(H_{S}\right)$, and fixation index $(F)$ were estimated in Powermarker v3.25 (Liu and Muse, 2005). Genetic distances among countries were calculated in Microsatellite Analyzer (MSA) v4.05 (Dieringer and Schlötterer, 2003), and cluster analyses on the basis of these distances were performed using PHYLIP v3.67 (Felsenstein, 2004) with the neighbor joining (NJ) method. Each branch was tested for reliability by re-sampling with 1000 replications. Finally, the NJ tree was viewed and edited in FigTree v1.4.2 (Rambaut, 2008). Allelic richness was determined by rarefaction analysis in HP-RARE v1.0, a program that 
compensates for sampling disparity among populations to allow comparison (Kalinowski, 2005). NewHybrids v1.1 beta (Anderson and Thompson, 2002) was used to classify hybrids into their respective classes as detailed in Wambulwa et al. (2016).

We carried out a model-based clustering analysis in STRUCTURE v2.3.4 (Pritchard et al., 2000) to estimate the number of genetic clusters $(K)$ without a priori knowledge of taxonomy or population location. STRUCTURE was run under the admixture model and correlated allele frequencies with 100,000 generations of "burn-in" and 100,000 Markov chain Monte Carlo (MCMC) iterations for increasing numbers of $K$ subdivisions from 1 to 8 . Simulations were repeated 20 times for each value of $K$. The optimal number of clusters $(K)$ was determined using both $\mathrm{L}(K)$ (Rosenberg et al., 2001) and $\Delta K$ (Evanno et al., 2005) methods visualized with STRUCTURE HARVESTER v0.6.92 (Earl and vonHoldt, 2012). A principal co-ordinates analysis (PCoA) was carried out in GenAIEx v6.5 (Peakall and Smouse, 2012).

\section{cpDNA Sequencing Data}

DNA sequences were assembled and edited in Sequencher v5.0 (Gene Codes Co., USA). The sequences were aligned with MUSCLE (Edgar, 2004). The edited sequences of the three cpDNA regions were then combined in SequenceMatrix v1.7.8 (Vaidya et al., 2011). Haplotypes of cpDNA sequences were defined using DnaSP v5.10 (Librado and Rozas, 2009), and a haplotype network tree constructed in NETWORK v4.6.1.3 (Fluxus Technology Ltd., Suffolk, UK) with the median-joining method (Bandelt et al., 1999). A Neighbor-joining tree of the 84 accessions was constructed in MEGA v6.0 (Tamura et al., 2013) based on Kimura 2-parameter (K2P) distances and a pairwise deletion model. Geographical mapping of the nSSR genetic groups and haplotype distribution was done sequentially in DIVA-GIS v7.5.0 (http://www.diva-gis.org/) and ArcGIS v10.2.2 (https://www.arcgis.com).

\section{RESULTS}

\section{Genetic Diversity of nSSR Markers}

No genotyping errors were detected in the nSSR dataset by MICROCHECKER. Only $0.34 \%$ missing data were present in the whole dataset due to consistent failures of PCR amplification. After rarefaction analysis, the mean number of alleles per locus across countries varied from 1.59 (TUGMS2 157) to 7.83 (Po9) (Table S3). Allelic richness ranged from 4.04 (Nigeria) to 6.6 (Cameroon). The overall genetic diversity $\left(H_{\mathrm{S}}\right)$ of the African tea accessions was 0.652 . At the country level, Kenya exhibited the highest genetic diversity $\left(H_{\mathrm{S}}=0.747\right)$, followed by Cameroon (0.716), and Rwanda (0.683), with South Africa having the lowest value (0.579). The fixation index $(F)$ for the eight countries ranged from -0.17 (South Africa) to 0.112 (Kenya). The summary statistics of the genetic indices are summarized in Table 1.
TABLE 1 | Summary statistics of genetic variation of the 23 SSR loci for 280 tea samples collected from eight African countries.

\begin{tabular}{lccccc}
\hline Country & $\boldsymbol{N}$ & $\boldsymbol{A}_{\mathbf{r}}$ & $\boldsymbol{H}_{\mathbf{O}}$ & $\boldsymbol{H}_{\mathbf{S}}$ & $\boldsymbol{F}$ \\
\hline Cameroon (CR) & 13 & 6.6 & 0.686 & 0.716 & 0.058 \\
Kenya (KN) & 183 & 6.49 & 0.663 & 0.747 & 0.112 \\
Madagascar (MD) & 15 & 4.42 & 0.556 & 0.614 & 0.097 \\
Malawi (MW) & 20 & 5.06 & 0.726 & 0.642 & -0.063 \\
Nigeria (NG) & 4 & 4.04 & 0.652 & 0.621 & -0.103 \\
Rwanda (RD) & 27 & 5.65 & 0.739 & 0.683 & -0.063 \\
South Africa (SA) & 8 & 4.74 & 0.630 & 0.579 & -0.170 \\
Tanzania (TZ) & 10 & 4.87 & 0.700 & 0.609 & -0.152 \\
\hline Total/Mean & 280 & & 0.6691 & 0.652 & -0.036
\end{tabular}

$N$, Sample size; $A_{r}$, Average allelic richness over loci; $H_{O}$, Observed heterozygosity; $H_{S}$, Genetic diversity; F, Fixation index.

\section{Genetic Structure and Clustering Patterns of nSSR Data}

The optimum value for $K$ was 2 , suggested by both $\mathrm{L}(K)$ and $\Delta K$ approaches (Figure S2). The genetic clustering of the 280 tea accessions is shown for $K=2,3$, and 4 (Figure 1). At $K$ $=2$, two genetic groups were defined, which corresponded to the $C$. sinensis var. sinensis and C. sinensis var. assamica groups found in our previous study (Wambulwa et al., 2016). Camellia sinensis var. sinensis (in red) included samples from Madagascar and some accessions from Kenya, while C. sinensis var. assamica (in green) contained most accessions from Kenya and those from the remaining six countries. Within both groups, many accessions possessed a mix of genetic material from both groups (representing "mosaic" genotypes). At $K=3$, a new group (in blue) was separated from the $C$. sinensis var. assamica group, and included accessions mainly from Malawi, South Africa, and Kenya, while overall more accessions showed a "mosaic" genotype. At $K=4$, some accessions from Kenya formed an additional group (in yellow) separated from the red group $(C$. sinensis var. sinensis; Figure 1). Geographical mapping of the genetic groups was based on the posterior probabilities at $K$ $=3$, because at this $K$-value, $C$. sinensis var. assamica (which constituted the majority of African tea) separated into two groups which are mainly distributed in southern and East Africa respectively (Figure 2 ).

The NJ tree showed three main clusters (Figure S1). The basal cluster (Cluster I) was comprised of seven accessions from Kenya (TRFK 91/1, TRFK 91/2, TRFK 306/1, TRFK 306/2, TRFK $306 / 3$, TRFK 306/4, and TRFK 830/5). Cluster II included three sub-clusters that contained a total of eleven accessions from Malawi, Kenya, Nigeria, and South Africa. The pioneer accession for the introduction of the tea plant in Malawi (SFS 150) and its counterparts in Kenya and South Africa formed one of the sub-clusters. The third cluster (Cluster III) consisted of the remaining 262 accessions which were grouped into 34 polytomic sub-clusters.

The clustering behavior of tea accessions in the PCoA plot highlighted the distinction between the southern and East African gene pools, a pattern that was generally consistent 

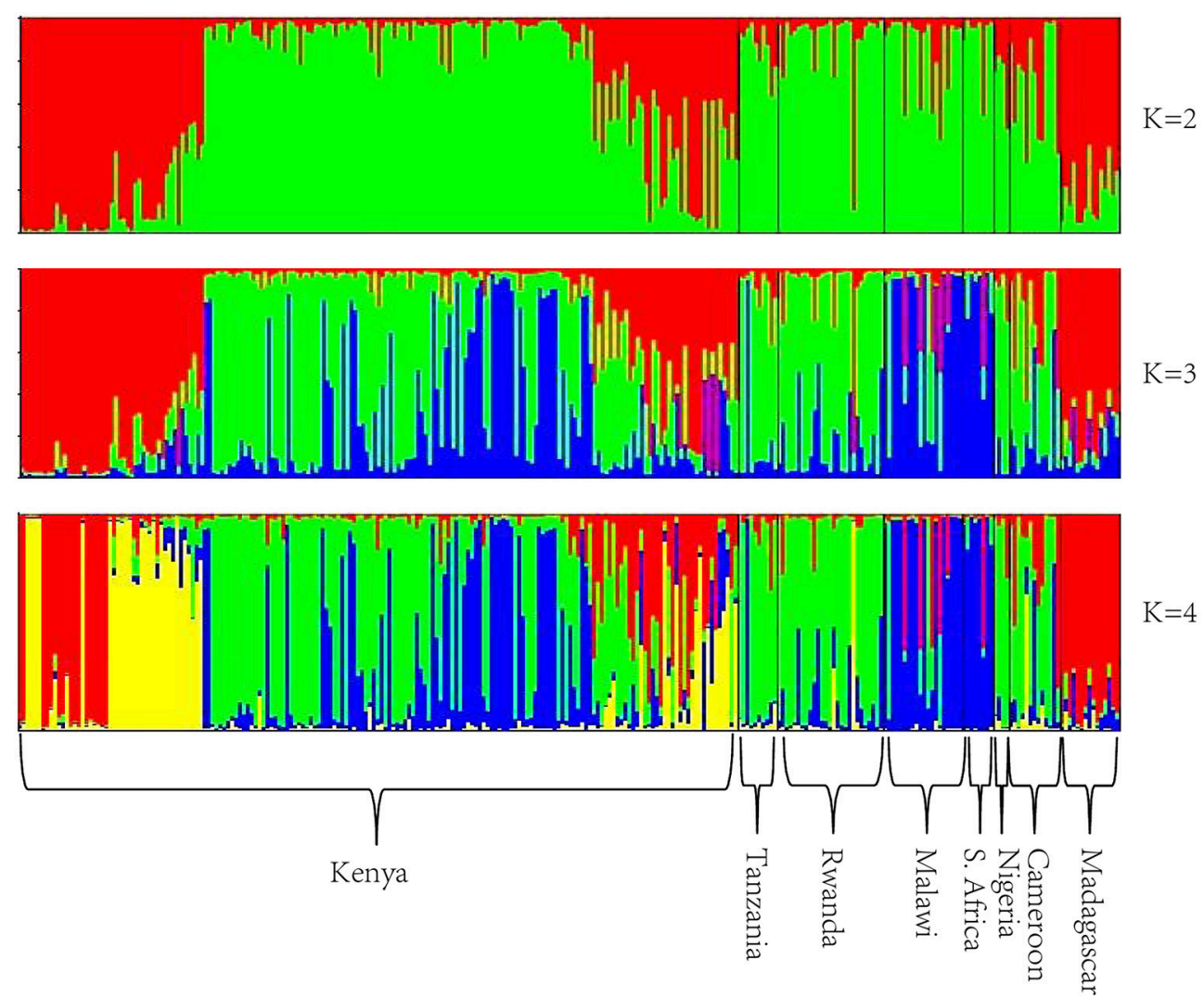

FIGURE 1 | Bayesian assignment of probabilities using STRUCTURE based on 23 nSSR loci of the 280 tea accessions.

with the NJ tree results (Figures S1, S3). Most accessions from Malawi and South Africa grouped into a single cluster including three accessions from Rwanda (35/49, 22/17, and K/32) and one from Tanzania (TRIT 201/82). The Kenyan accessions, TRFK $31 / 11$ and TRFK 18/16, formed a single cluster together with 10 accessions from Rwanda. Five Tanzanian tea accessions grouped together with three Kenyan accessions (TRFK 430/90, TRFK 420/13, and TRFK 14/1). Some Rwandan accessions, e.g., RW/G539, RW/SR/2B1, and RW/SR/2B1/49, clustered separately with Kenyan accessions.

In fact, no clear country-specific genetic cluster was observed, except perhaps for Madagascar, albeit this including one accession from Cameroon (CL H81/22). In addition, in six cases accessions from different countries but with similar codes fell in the same subclusters: PC 108 (Malawi) and PC 108, 15/4PC 108 (South Africa); PC 81 (Malawi), 16/4PC 81 (South Africa), and AHP PC 81 (Kenya); SFS 150 (Malawi), 16/4SFS 150 (South Africa), and TRFCA SFS 150 (Kenya); BBK 35 (Kenya) and Var BB 35 (Nigeria); TRFK 6/8 (Kenya) and Var 68 (Nigeria); and SFS 204 (Malawi) and 16/4SFS 204 (South Africa).

In the NewHybrids analysis, a total of 22 accessions from Kenya were assigned P1 (parent 1), and 146 accessions assigned P2 (parent 2). The two parent assignations corresponded to $C$. sinensis var. sinensis and C. sinensis var. assamica, respectively, (Figure 3). For the remaining 112 accessions, a hybrid origin was suggested, including $70 \mathrm{~F} 2$ s (second filial generation), one BC1 (backcross to parent 1), and $41 \mathrm{BC} 2 \mathrm{~s}$ (backcross to parent 2), but no F1 (first filial generation) generation hybrid was detected. All 15 samples from Madagascar were assigned F2 hybrids status. Details of the distribution of the various parental and hybrid classes are provided in Tables S1, S4.

\section{cpDNA Sequencing and Relationships of Haplotypes}

The alignment of the three cpDNA regions of $n d h F-r p l 32$, trnSGG-trnSr, and trnSf1-trnGGG was $661 \mathrm{bp}, 647 \mathrm{bp}$, and $601 \mathrm{bp}$ in length, respectively. The concatenated matrix of the three cpDNA regions was 1909 bp long and contained 42 polymorphic sites $(2.2 \%)$. A total of nine haplotypes $(\mathrm{H} 1-\mathrm{H} 9)$ were identified for the 84 African tea accessions. Four out of these were private to particular countries, two found in Kenya, and one each in Cameroon and Madagascar, respectively. The Kenyan samples had the highest number of haplotypes (7), followed by Cameroon (4) then Rwanda (3) (Figure 4, Table S5). The haplotype network and NJ tree revealed similar relationships (Figures 5, 6). The nine haplotypes could be divided into two clades. Haplotypes $\mathrm{H} 1$, $\mathrm{H} 2, \mathrm{H} 6$, and $\mathrm{H} 8$ formed Clade 1 , which was associated with $C$. sinensis var. assamica. In this clade, $\mathrm{H} 1$ and $\mathrm{H} 2$ were the two most dominant haplotype with one mutation step between them, with $\mathrm{H} 1$ mainly occurring in East and West Africa, while $\mathrm{H} 2$ was dominant in southern Africa. Clade 2 consisted of the remaining haplotypes and was associated with C. sinensis var. sinensis; H3 was specific to $C$. sinensis var. sinensis while $\mathrm{H} 9$ was present in 


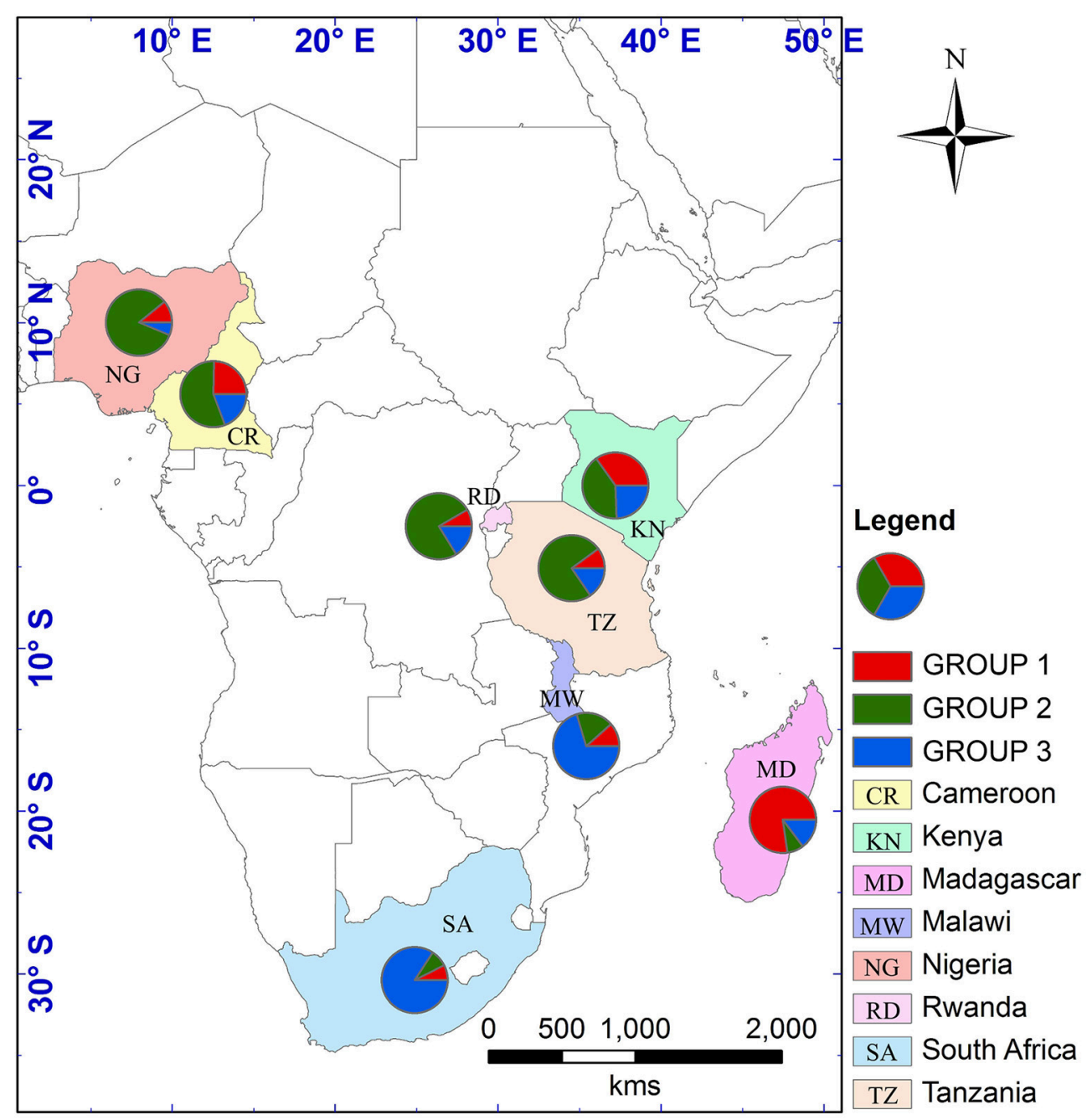

FIGURE 2 | Geographical distribution of nSSR genetic groups considered following STRUCTURE analysis at $\boldsymbol{K}=\mathbf{3}$. Each country is depicted as a pie chart with the proportional membership of its alleles to each one of the three groups. A shape file with genotype proportions in the different countries was generated in DIVA-GIS v7.5.0.0 (http://www. diva-gis.org/). The shape file was then used to generate the map in ArcGIS v10.2.2 (http://www.esri.com/).

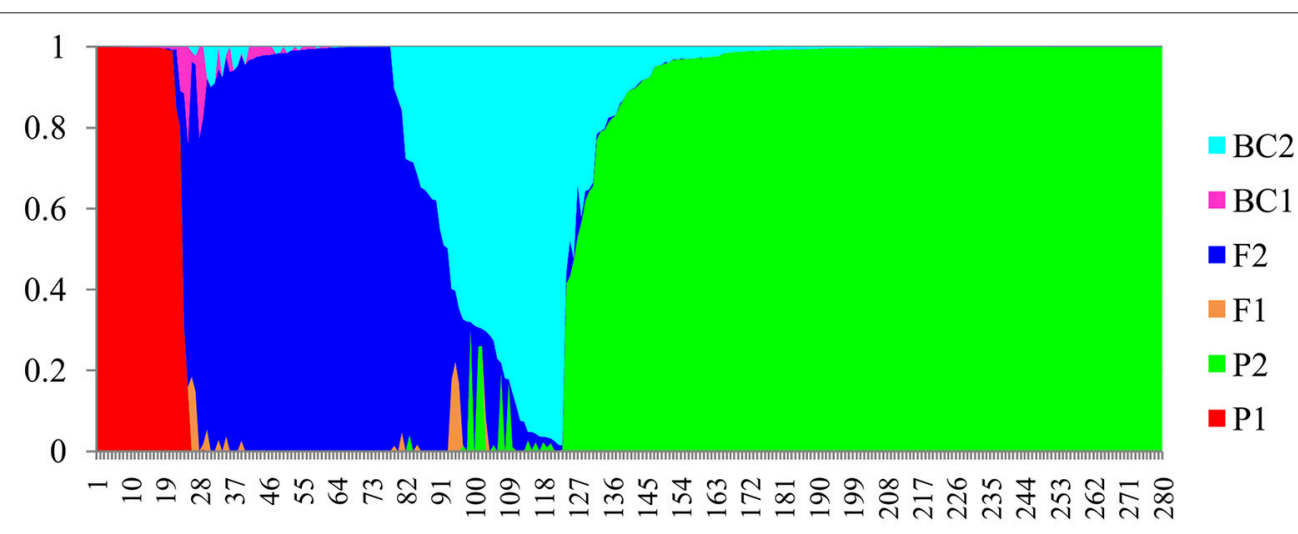

FIGURE 3 | Bayesian assignment of probabilities in NewHybrids for $\mathbf{2 8 0}$ tea accessions from Africa. The defined categories are parent 1 (P1), parent 2 (P2), first filial generation (F1), second filial generation (F2), backcross to P1 (BC1), and backcross to P2 (BC2). 


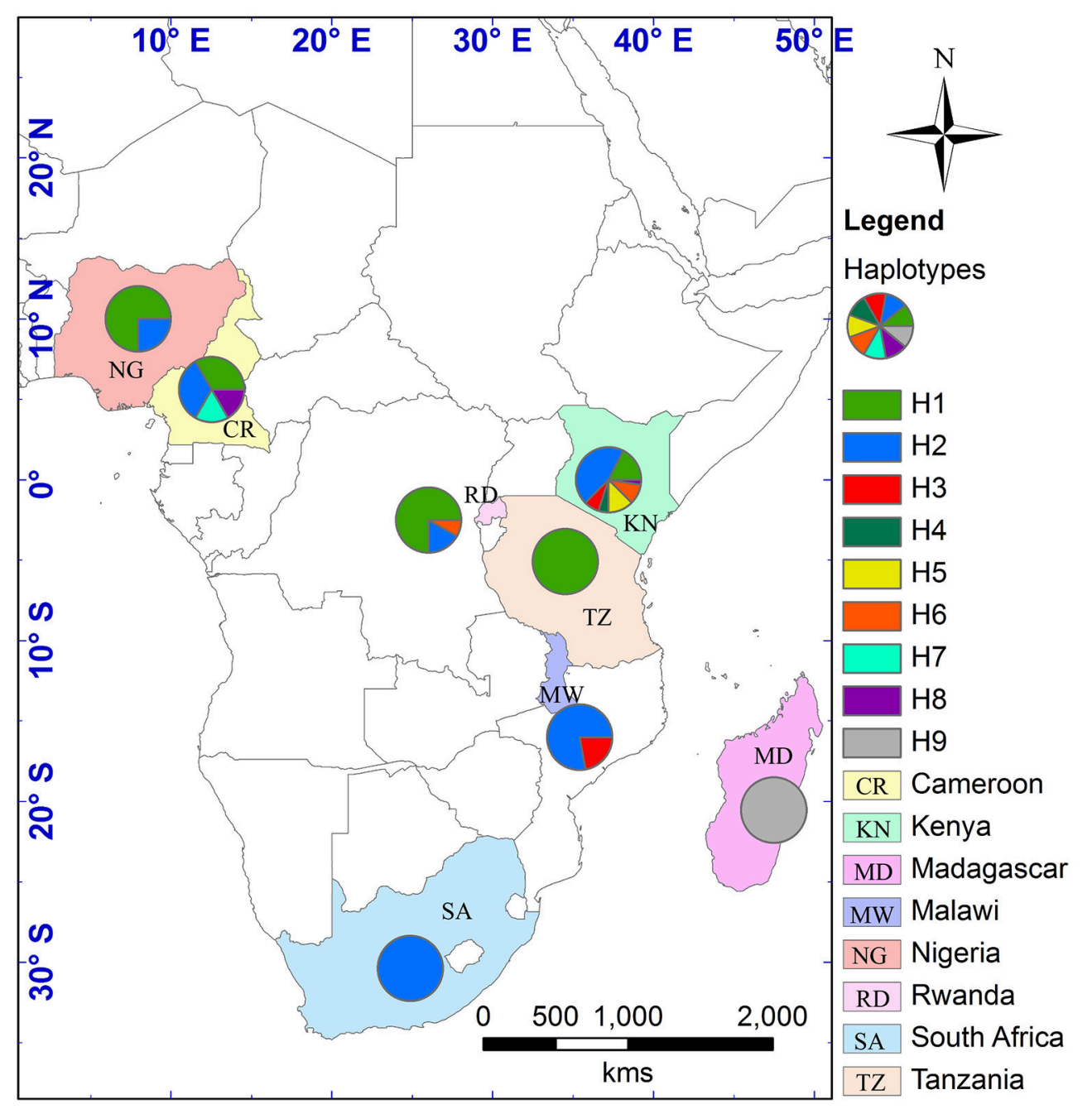

FIGURE 4 | Geographical distribution of the nine cpDNA found in the $\mathbf{2 8 0}$ samples across eight countries in Africa. The map was generated using DIVA-GIS and ArcGIS software as described in Figure 2.

all samples from Madagascar, corresponding to C. sinensis var. pubilimba. Haplotypes $\mathrm{H} 4, \mathrm{H} 5$, and $\mathrm{H} 7$ may represent a distinct lineage of tea; for instance $\mathrm{H} 4$ is shared with accession TRFK 91/1 which is Camellia irrawadiensis, a wild relative of the tea plant. Overall, $\mathrm{H} 1$ and $\mathrm{H} 2$ showed the highest proportions among all accessions with 32.14 and $40.48 \%$, respectively. Comparison with the NewHybrids results showed that all accessions in Clade 1 (except TRFK 306/4) were of P2, F2, and BC2 origin, while all accessions with $\mathrm{H} 3$ and $\mathrm{H} 9$ were assigned P1 and F2 (H9) status.

The accessions CL 671 and China Hybrid Seedling fell in one subcluster based on the nSSR data (Figure S1), but had different cpDNA haplotypes of $\mathrm{H} 2$, and $\mathrm{H} 3$, respectively, (Table S5). TRFK 430/90 and TRIT 201/75, and CL H81/22 and the Madagascan accessions, showed the same pattern. On the other hand, the three known hybrids between TRFCA SFS 150 and EPK TN143 (TRFK 430/52, TRFK 430/63, and TRFK 430/90) from Kenya shared the same haplotype with SFS 150.

\section{DISCUSSION}

\section{Genetic Diversity and Clustering}

Genotyping and assessment of genetic diversity is an important prerequisite for the identification of core germplasm collections for conservation and optimizing crop improvement efforts (Fu, 2015). This is the first study to assess the genetic diversity of the tea plant across all the main tea-growing countries in Africa. We found that the genetic diversity of African tea (0.652) is much lower than that of Asia (0.86) calculated over 788 accessions from 14 countries, with both studies using 23 nSSR markers (Taniguchi et al., 2014). The higher diversity estimated for this region possibly results from the wider genetic sampling from the cradle of the tea plant in China and India (Hashimoto and Takashi, 1978; Hashimoto, 2001), which inherently possess higher levels of genetic diversity (Yao et al., 2012). Interestingly, our recent study found higher genetic variation among the East African tea accessions (0.717), probably 


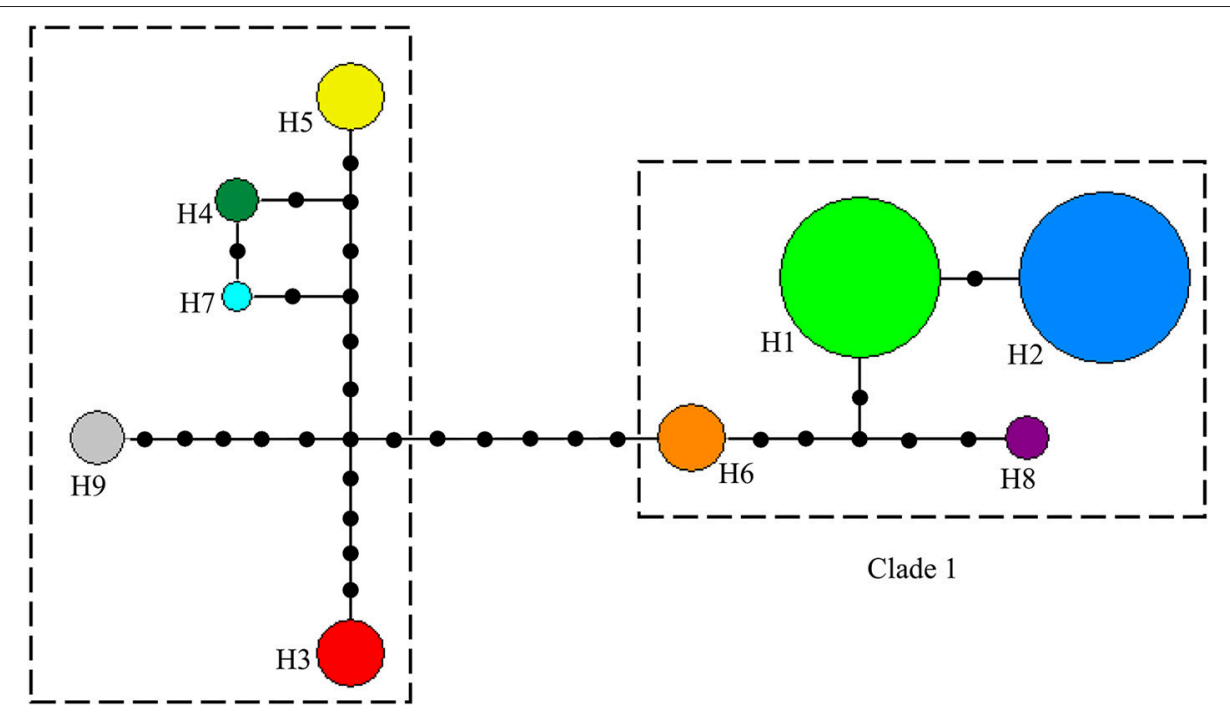

Clade 2

FIGURE 5 | Median-joining haplotype network based on three combined cpDNA regions of 84 tea samples from eight African countries. Broken lines delineate closely related haplotypes. Circles in colors denote different haplotypes $(\mathrm{H} 1-\mathrm{H} 9)$, and the size of each circle is proportional to the number of accessions sharing that particular haplotype. Each branch between haplotypes denotes a mutational step. The small black circles represent independent mutation events converging on a shared haplotype.

because of the ex situ germplasm bank of tea plants in Kenya which contains accessions that are genetically highly disparate and assembled from various geographical origins (Wambulwa et al., 2016).

In a separate study, however, the Kenyan tea samples harbored the lowest genetic diversity when compared to Chinese and Japanese teas (Yao et al., 2008). The low variation could be attributed to the relatively short history of Kenyan tea plantations, the small sample size (only four accessions included) and the dominant markers used (ISSR). Sample size bias may be a potential confounding factor of genetic diversity estimates. In this study, we determined allelic richness based on rarefaction analysis, thus eliminating the problem of sample size disparity. Cameroon also showed the highest allelic richness $\left(A_{\mathrm{r}}=6.6\right)$ despite its relatively small sample size, which possibly resulted from the diverse genetic origin of the tea cultivars in this country, as evidenced by the existence of five haplotypes including a private one. Unsurprisingly, among the African accessions, Kenya also exhibited a high genetic variation perhaps due to the diversity of geographic origins of the samples (Wambulwa et al., 2016).

Our results highlight the importance of the combination of differently inherited markers to clarify the breeding patterns of the tea plants in Africa. For example, TRFK 430/52, TRFK 430/63, and TRFK 430/90 are known to be hybrids between TRFCA SFS150 and EPK TN14-3 but without information on the direction of the cross. The haplotype analysis identified TRFCA SFS 150 to be the maternal parent, because the three hybrids shared its haplotype. In addition, Madagascan tea accessions could not be separated from $C$. sinensis var. sinensis accessions from Kenya by nSSR data. However, the cpDNA data could separate them into distinct haplotypes $\mathrm{H} 3$ and $\mathrm{H} 9$. This might indicate that the tea cultivars from Madagascar are of likely hybrid origin between C. sinensis var. sinensis (paternal) and C. sinensis var. pubilimba (maternal) native to South China, and probably introduced independently of those from mainland Africa. Accessions CL671 and China Hybrid Seedling also have different haplotypes but a similar nuclear genetic composition, implying that these accessions are also hybrids of BC or F2 (or later) generation with diverse maternal parents but probably sharing the same paternal parent, which was confirmed in our NewHybrids analysis.

\section{Genetic Relationships and Germplasm Exchange of Tea Accessions within Africa}

Our results showed that African tea germplasm is genetically admixed and shared among countries, indicating that the tea accessions were often exchanged among different countries. For instance, the clustering patterns in the NJ tree suggested that the Rwandan accessions $\mathrm{RW} / 35 / 49, \mathrm{RW} / 22 / 17$, and $\mathrm{RW} / \mathrm{K} / 32$ and the Tanzanian accession TRIT 201/82 might have been introduced from South Africa, while the Kenyan accessions TRFK 430/90, TRFK 420/13, and TRFK 14/1 possibly originated from Tanzania. The typically southern accessions of the SFS and PC series (with haplotype $\mathrm{H} 2$ ) are dominant in southern Africa and with low frequency in Kenya, Rwanda, Cameroon, and Nigeria, suggesting germplasm movement from the southern Africa region (Malawi and South Africa) northwards. Moreover, historical records showed that the southern breeding program (TRFCA) is the oldest in Africa (Anonymous, 1962). We therefore deduced that some of the pioneer germplasm or offspring thereof might have been moved northwards from 


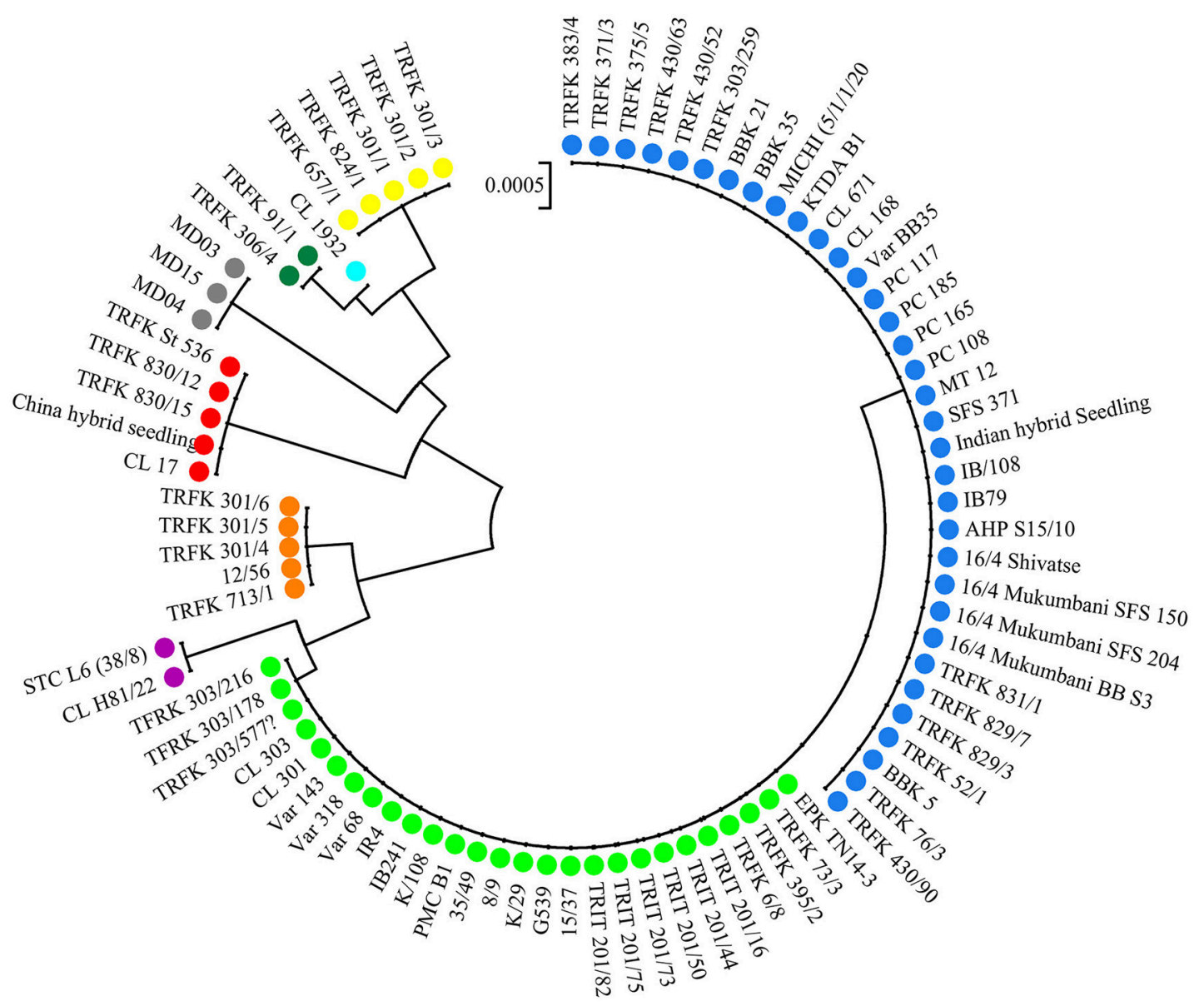

FIGURE 6 | Neighbor joining tree of $\mathbf{8 4}$ tea samples. Each color represents a particular haplotype and corresponds to colors in Figure $\mathbf{5}$.

southern Africa to East Africa and eventually to West Africa. Unique haplotypes were found in Cameroon, Kenya, and Madagascar, which suggested possibly independent germplasm exchanges and introductions from countries outside of Africa. Germplasm exchanges among countries may be an important strategy for tea or crop improvement in general. International exchange of crop germplasm is essential both for ensuring food security and for promoting research relations among the countries involved (Singh et al., 2012). In the face of the threats from climate change, interdependency among countries for genetic material of crop plants is expected to accelerate the development of new varieties for better adaptation to future climate challenges (FAO, 2015b). However, germplasm exchange should be exercised cautiously and aided by genetic fingerprinting methods, or otherwise problems of nominal redundancies in the accession databases may arise, exacerbated by the tendency of some countries to adopt different names for the same accession (Kisha and Cramer, 2011). In the present study, we did indeed find that accessions with similar names from different countries clustered together (e.g., SFS 150 from Malawi, 16/4SFS 150 from South Africa, and TRFCA SFS 150 from Kenya), suggesting that they are the same accession with slightly modified identifiers.

The NewHybrids analysis here showed similar results to our previous study on East African tea accessions (Wambulwa et al., 2016). Cultivars of C. sinensis var. assamica are the most popular and dominant tea plants in Africa. The popularity of C. sinensis var. assamica might be linked to its faster growth, ease of harvest and higher yields (Seurei, 1996). Accessions of C. sinensis var. sinensis on the other hand, are mainly held in germplasm banks as a breeding resource for hybridization, due to their lower yield because of the small leaves that are difficult to pluck. The introduction of machine harvesting, however, may result in commercialization of some of these cultivars for green and specialty tea processing in the future. Hybrid cultivars made up a high proportion of 62.5 (F2) and $36.6 \%$ (BC2) of the accessions. In these accessions, $C$. sinensis var. assamica contributed much more than $C$. sinensis var. sinensis as maternal parent. The distinct genetic group formed by the hybrids (Figure 1, in yellow) indicated that these cultivars are possibly fixed for some alleles linked to adaptation to local environments during their breeding history. 


\section{Breeding Centers and Conservation of Tea Germplasm}

Southern Africa (TRFCA) and East Africa (TRFK) are recognized as the oldest breeding centers in Africa (Matheson and Bovill, 1950; Anonymous, 1962). Our results of both nSSR and cpDNA haplotype analysis basically reflect the activities of the two breeding centers on mainland Africa. Two distinct genetic groups were defined for C. sinensis var. assamica accessions, a southern group (in blue) and an eastern group (in green; Figures 2, 4). Although the haplotypes $\mathrm{H} 1$ and $\mathrm{H} 2$ differed only by a single mutation, $\mathrm{H} 1$ was associated with the pioneer selection TRFK 6/8 from East Africa, and $\mathrm{H} 2$ is preponderant in pioneer selections SFS 371, SFS 150, and SFS 204 from southern Africa (South Africa and Malawi; Figure 4). We concluded that TRFCA (southern Africa) and TRFK (East Africa) are the two main tea breeding centers for $C$. sinensis var. assamica in Africa, and have independently selected cultivars for specific traits. The occurrence of two distinct gene pools of $C$. sinensis var. assamica could be attributed to breeders preferences and the subsequent accumulation of divergently selected traits from distinct genetic resources to breed new preeminent cultivars.

Yield and quality are the universal traits of focus in tea breeding (Mondal, 2014). However, climate adaptationassociated traits might have played an important role in differentiating the accessions of the two breeding centers. Such traits include drought tolerance, reduced winter dormancy, hail/frost resistance, water logging tolerance, and cold hardiness. Southern Africa has a transition zone of sub-tropical and temperate climates, with desert or semi-arid regions centered in Namibia and Botswana, while eastern (equatorial) Africa has a tropical climate with a relatively high altitude and high annual precipitation. Recent studies on the tea plant have demonstrated that variations in climatic conditions can significantly affect profiles of secondary metabolite such as catechins and methylxanthines (Ahmed et al., 2014; Kowalsick et al., 2014; Larson, 2015) which directly influence tea quality. Going forward, it will be important for tea breeders to make use of niche modeling and climate data as a novel strategy for developing locally well adapted tea cultivars from divergent gene pools.

Camellia sinensis var. assamica is the main tea type under cultivation in Africa, a situation that may compromise the ability of the germplasm to adapt to biotic and abiotic challenges as a result of its relative genetic homogeneity. It is therefore important to maintain diverse genetic germplasm lineages. In the present study, several divergent haplotypes $(\mathrm{H} 4, \mathrm{H} 5, \mathrm{H} 7$, and H9) not derived from the tea plant were observed in the African germplasm, and may represent distinct gene pools, such as C. sinensis var. assamica in China that was recently defined as a new genetic entity (Meegahakumbura et al., 2016), and wild relatives of the tea plant. In fact, $\mathrm{H} 4$ represented a haplotype derived from C. irrawadiensis, native to Myanmar, which might have been introduced to breed for the presence of anthocyanin (purple) pigments in certain tea accessions (Kamunya et al., 2009). These accessions may be highly valuable resources for tea breeding in the future. Thus, it is very important to conserve such elite genetic stock as resources diversifying the African tea gene pool to be flexible to adjust to future directions in tea breeding. The phenomenon of differential selection by breeders is not limited to our study. A similar observation was made in maize (Van Heerwaarden et al., 2012), sorghum (Morris et al., 2013), wheat (Cavanagh et al., 2013), and rice (Xie et al., 2015) where accessions originating from different breeding programmes showed high differentiation. It has been shown that locally selected material collectively possesses a high diversity and potential for local adaptation (Ravigné et al., 2009), which will be very important for mitigating the adverse influence of climate change and other environmental abiotic and biotic challenges.

\section{CONCLUSIONS}

We investigated the genetic diversity and relationships for 280 African tea accessions based on 23 polymorphic nSSR markers and three cpDNA sequence regions. Our results indicated that African tea has a relatively low genetic diversity, and $C$. sinensis var. assamica is the main tea type under cultivation and contributes more in tea breeding improvement programs in Africa. Internal germplasm exchange and movement among countries within Africa is evident, with Southern Africa (TRFCA) and East Africa (TRFK) possibly being the two major tea breeding centers in Africa. This study represents the first step toward effective utilization of differently inherited molecular markers for exploring the breeding improvement of African tea. The findings will be important for planning the exploration, utilization, and conservation of tea germplasm for future breeding efforts in Africa.

\section{DATA ACCESSIBILITY}

The nSSR data set supporting the results in this study are available from the Dryad Digital Repository: http://dx.doi.org/ $10.5061 /$ dryad.3g02j. The cpDNA sequence data in this study are deposited in GenBank under accession numbers KX584101KX584352 as shown in Table S1.

\section{AUTHOR CONTRIBUTIONS}

MW, SK, and AM collected plant material. MW and MKM performed the experiments. MW, MM, SR, JL, and LG analyzed and interpreted the data. MW, SK, MM, DL, and LG wrote and revised the manuscript. DL, LG, JX, and AM conceived and designed the study. All authors reviewed and approved the final manuscript.

\section{ACKNOWLEDGMENTS}

We thank Miao-Miao Li, Jun-Bo Yang, Hong-Tao Li, Jing Yang, Zhi-Rong Zhang and Shi-Xiong Yang for their support of laboratory work and specimen identification, and Josephine Makueti, Oluwatoyin Ogundipe and Ting Zhang for their help 
with sample collection. This work was funded by the National Natural Science Foundation of China (31161140350). We also acknowledge the CGIAR CRP 6.2 program "Tree diversity and domestication" for their technical input on the manuscript. The Royal Botanic Garden Edinburgh is funded by the Rural and Environment Science and Analytical Services Division (RESAS) of the Scottish Government. Laboratory work was performed at the Molecular Biology Centre of the Germplasm Bank of
Wild Species, Kunming Institute of Botany, Chinese Academy of Sciences.

\section{SUPPLEMENTARY MATERIAL}

The Supplementary Material for this article can be found online at: http://journal.frontiersin.org/article/10.3389/fpls.2016. 01244

\section{REFERENCES}

Ahmed, S., Stepp, J. R., Orians, C., Griffin, T., Matyas, C., Robbat, A., et al. (2014). Effects of extreme climate events on tea (Camellia sinensis) functional quality validate indigenous farmer knowledge and sensory preferences in tropical China. PLoS ONE 9:e109126. doi: 10.1371/journal.pone. 0109126

Allan, G. J., and Max, T. L. (2010). Molecular genetic techniques and markers for ecological research. Nat. Educ. Knowl. 3, 2.

Anderson, E. C., and Thompson, E. A. (2002). A model-based method for identifying species hybrids using multilocus genetic data. Genetics 160, 1217-1229.

Anonymous. (1962). "Historical notes on tea introduction in Africa," in Tea Estates in Africa, ed S. Wilson (London: Mabey \& Fitzclarence), 6-9.

Bandelt, H. J., Forster, P., and Röhl, A. (1999). Median-joining networks for inferring intraspecific phylogenies. Mol. Biol. Evol. 16, 37-48. doi: 10.1093/oxfordjournals.molbev.a026036

Bedran, T. B. L., Morin, M. P., Spolidorio, D. P., and Grenier, D. (2015). Black tea extract and its theaflavin derivatives inhibit the growth of periodontopathogens and modulate interleukin-8 and $\beta$-defensin secretion in oral epithelial cells. PLoS ONE 10:e0143158. doi: 10.1371/journal.pone.0143158

Cavanagh, C. R., Chao, S., Wang, S., Huang, B. E., Stephen, S., Kiani, S., et al. (2013). Genome-wide comparative diversity uncovers multiple targets of selection for improvement in hexaploid wheat landraces and cultivars. Proc. Natl. Acad. Sci. U.S.A. 110, 8057-8062. doi: 10.1073/pnas.12171 33110

Chase, M., Kesseli, R., and Bawa, K. (1996). Microsatellite markers for conservation and population genetics of tropical tree species. Am. J. Bot. 83, 51-57. doi: $10.2307 / 2445953$

Clegg, M. T., and Zurawski, G. (1992). "Chloroplast DNA and the study of plant phylogenetics: present status and future prospects," in Molecular Systematics of Plants, eds P. S. Soltis, D. E. Soltis, and J. J. Doyle (New York, NY: Chapman \& Hall), 1-13.

Dieringer, D., and Schlötterer, C. (2003). Microsatellite analyser (MSA): a platform independent analysis tool for large microsatellite data sets. Mol. Ecol. Notes 3, 167-169. doi: 10.1046/j.1471-8286.2003.00351.x

Doyle, J. J., and Doyle, J. L. (1990). Isolation of plant DNA from fresh tissue. Focus $12,13-15$.

Earl, D. A., and vonHoldt, B. M. (2012). STRUCTURE HARVESTER: a website and program for visualizing STRUCTURE output and implementing the Evanno method. Conserv. Genet. Resour. 4, 359-361. doi: 10.1007/s12686-011-9548-7

Edgar, R. C. (2004). MUSCLE: multiple sequence alignment with high accuracy and high throughput. Nucleic Acids Res. 32, 1792-1797. doi: $10.1093 / \mathrm{nar} / \mathrm{gkh} 340$

Ellegren, H. (2004). Microsatellites: simple sequences with complex evolution. Nat. Rev. Genet. 5, 435-445. doi: 10.1038/nrg1348

Ellis, R. T., and Nyirenda, H. E. (1995). A successful plant improvement programme on tea (Camellia sinensis). Expt. Agric. 31, 307-323. doi: 10.1017/ S0014479700025485

Evanno, G., Regnault, S., and Goudet, J. (2005). Detecting the number of clusters of individuals using the software structure. A simulation study. Mol. Ecol. 14, 2611-2620. doi: 10.1111/j.1365-294X.2005.02553.x

FAO(Food and Agricultural Organization of the United Nations). (2014). Committee on Commodity Problems. Rome: Food and Agricultural Organization of the United Nations. 25.

FAO (Food and Agricultural Organization of the United Nations). (2015a). Contribution of Tea Production and Exports to Food Security, Rural Development and Smallholder Welfare in Selected Producing Countries. Rome: Food and Agricultural Organization of the United Nations.

FAO (Food and Agricultural Organization of the United Nations). (2015b). Coping with Climate Change - the Roles of Genetic Resources for Food and Agriculture. Rome: Food and Agricultural Organization of the United Nations.

FAOSTAT. (2015). FAO database. Food Agric. Organ. United Nations. Available online at: http://faostat3.fao.org/download/Q/QC/E (Accessed 10 May 2015).

Fan, L., Zhang, M. Y., Liu, Q. Z., Li, L. T., Song, Y., Wang, L. F., et al. (2013). Transferability of newly developed pear SSR markers to other Rosaceae species. Plant. Mol. Biol. Rep. 31, 1271-1282. doi: 10.1007/s11105-013-0586-Z

Felsenstein, J. (2004). PHYLIP (Phylogeny Inference Package) version 3.6. Distributed by the author. Department of Genome Sciences, Seattle: University of Washington.

Fu, Y. B. (2015). Understanding crop genetic diversity under modern plant breeding. Theor. Appl. Genet. 128, 2131-2142. doi: 10.1007/s00122-015-2585-y

Hashimoto, M. (2001). "The Origin of the Tea Plant," in Proceedings of 2001 International Conference on O-CHA (tea) Culture and Science (Session II) (Shizuoka).

Hashimoto, M., and Takashi, S. (1978). Morphological studies on the origin of the tea plant $\mathrm{V}$, a proposal of one place of origin by cluster analysis. Jap. J. Trop. Agric. 21, 93-101.

Kalinowski, S. T. (2005). HP-RARE 1.0: a computer program for performing rare faction on measures of allelic richness. Mol. Ecol. Notes 5, 187-189. doi: 10.1111/j.1471-8286.2004.00845.x

Kamunya, S. M., Wachira, F. N., Nyabundi, K. W., Kerio, L., and Chalo, R. M. (2009). The Tea Research Foundation of Kenya pre-releases purple tea variety for processing health tea product. Tea 30, 3-10.

Kaundun, S. S., and Matsumoto, S. (2011). Molecular evidence for maternal inheritance of the chloroplast genome in tea, Camellia sinensis (L.) O. Kuntze. J. Sci. Food. Agric. 91, 2660-2663. doi: 10.1002/jsfa.4508

Kisha, T. J., and Cramer, C. S. (2011). Determining redundancy of short-day onion accessions in a germplasm collection using microsatellite and targeted region amplified polymorphic markers. J. Am. Soc. Hort. Sci. 136, 129-134.

Kowalsick, A., Kfoury, N., Robbat, A. Jr., Ahmed, S., Orians, C., Griffin, T., et al. (2014). Metabolite profiling of Camellia sinensis by automated sequential, multidimensional gas chromatography/mass spectrometry reveals strong monsoon effects on tea constituents. J. Chrom. 1370, 230-239. doi: 10.1016/j.chroma.2014.10.058

Larson, C. (2015). Reading the tea leaves for effects of climate change. Science 348, 953-954. doi: 10.1126/science.348.6238.953

Librado, P., and Rozas, J. (2009). DnaSP v5: A software for comprehensive analysis of DNA polymorphism data. Bioinformatics 25, 1451-1452. doi: 10.1093/bioinformatics/btp187

Liu, K., and Muse, S. V. (2005). PowerMaker: integrated analysis environment for genetic marker data. Bioinformatics 21, 2128-2129. doi: 10.1093/bioinformatics/bti282

Matheson, J. K., and Bovill, E. W. (1950). Tea. East African Agriculture: A Short Survey of the Agriculture of Kenya, Uganda, Tanganyika, and Zanzibar and of its Principal Products. London: Oxford University Press.

Meegahakumbura, M. K., Wambulwa, M. C., Thapa, K. K., Li, M. M., Möller, M., Xu, J. C., et al. (2016). Indications for three independent domestication events for the tea plant (Camellia sinensis (L.) O. Kuntze) and new insights into the origin of tea germplasm in China and India revealed by 
nuclear microsatellites. PLoS ONE 11:e0155369. doi: 10.1371/journal.pone.01 55369

Ming, T. L. (2000). Monograph of the Genus Camellia. Kunming: Yunnan Science and Technology Press.

Ming, T. L., and Bartholomew, B. (2007). “Theaceae," in Flora of China, eds Z. Y. Wu, P. H. Raven and D. Y. Hong (Beijing: Science Press and St. Louis: Missouri Botanical Garden Press), 366-478.

Mogensen, H. L. (1996). The hows and whys of cytoplasmic inheritance in seed plants. Amer. J. Bot. 83, 383-404. doi: 10.2307/2446172

Mondal, T. K. (2014). Breeding and Biotechnology of Tea and its Wild Species. New York, NY: Springer Science and Business Media.

Morris, G. P., Ramub, P., Deshpande, S. P., Hash, C. T., Shah, T., Upadhyaya, H. D., et al. (2013). Population genomic and genome-wide association studies of agro-climatic traits in sorghum. Proc. Natl. Acad. Sci. U.S.A. 110, 453-458. doi: 10.1073/pnas.1215985110

Paul, S., Wachira, F. N., Powell, W., and Waugh, R. (1997). Diversity and genetic differentiation among populations of Indian and Kenyan tea (Camellia sinensis (L.) O. Kuntze) revealed by AFLP markers. Theor. Appl. Genet. 94, 255-263. doi: $10.1007 / \mathrm{s} 001220050408$

Paule, J., Scherbantin, A., and Dobeš, C. (2012). Implications of hybridisation and cytotypic differentiation in speciation assessed by AFLP and plastid haplotypes - a case study of Potentilla alpicola La Soie. BMC Evol. Biol. 12:132. doi: 10.1186/1471-2148-12-132

Peakall, R., and Smouse, P. (2012). GenAlEx 6.5: genetic analysis in Excel. Population genetic software for teaching and research - an update. Bioinformatics 82, 2537-2539. doi: 10.1093/bioinformatics/bts460

Pritchard, J. K., Stephens, M., and Donnelly, P. (2000). Inference of population structure using multilocus genotype data. Genetics 155, 945-959.

Rambaut, A. (2008). FigTree, v1.3.1. Institute of Evolutionary Biology. University of Edinburgh. Available online at: http://tree.bio.ed.ac.uk/software/figtree/ (Accessed 27 October 2015).

Ravigné, V., Dieckmann, U., and Olivieri, I. (2009). Live where you thrive: joint evolution of habitat choice and local adaptation facilitates specialization and promotes diversity. Am. Nat. 174, E141-E169. doi: 10.1086/ 605369

Rosenberg, N. A., Burke, T., Elo, K., Feldman, M. W., Freidlin, P. J., Groenen, M. A., et al. (2001). Empirical evaluation of genetic clustering methods using multilocus genotypes from 20 chicken breeds. Genetics 159, 699-713.

Selkoe, K. A., and Toone, R. J. (2006). Microsatellites for ecologists: a practical guide to using and evaluating microsatellite markers. Ecol. Lett. 9, 615-629. doi: 10.1111/j.1461-0248.2006.00889.x

Seurei, P. (1996). Tea improvement in Kenya: a review. Tea 17, 76-81.

Singh, R. P., Rajaram, S., Miranda, A., Huerta-Espino, J., and Autrique, E. (2012). "Comparison of two crossing and four selection schemes for yield, yield traits and slow rusting resistance to leaf rust in wheat," in Wheat: Prospects for Global Improvement, eds H. J. Braun, F. Altay, W. E. Kronstad, S. P. S. Beniwal and A. McNab (New York, NY: Springer Science), 388.

Tamura, K., Stecher, G., Peterson, D., Filipski, A., and Kumar, S. (2013). MEGA6: Molecular Evolutionary Genetics Analysis version 6.0. Mol. Biol. Evol. 30, 2725-2729. doi: 10.1093/molbev/mst197

Taniguchi, F., Kimura, K., Saba, T., Ogino, A., Yamaguchi, S., and Tanaka, J. (2014). Worldwide core collections of tea (Camellia sinensis) based on SSR markers. Tree Genet. Genomes 10, 1555-1565. doi: 10.1007/s11295-014-0779-0

Vaidya, G., Lohman, D. J., and Meier, R. (2011). SequenceMatrix: Concatenation software for the fast assembly of multigene datasets with character set and codon information. Cladistics, 27, 171-180. doi: 10.1111/j.10960031.2010.00329.x
Van Heerwaarden, J., Hufford, M. B., and Ross-Ibarra, J. (2012). Historical genomics of North American maize. Proc. Natl. Acad. Sci. U.S.A. 109, 12420-12425. doi: 10.1073/pnas.1209275109

Van Oosterhout, C., Hutchinson, W. F., Wills, D. P. M., and Shipley, P. (2004). MICRO-CHECKER: software for identifying and correcting genotyping errors in microsatellite data. Mol. Ecol. Resour. 4, 535-538. doi: 10.1111/j.14718286.2004.00684.x

Wachira, F. N., Waugh, R., Hackett, C. A., and Powell, W. (1995). Detection of genetic diversity in tea (Camellia sinensis) using RAPD markers. Genome 38, 201-210. doi: 10.1139/g95-025

Wambulwa, M. C., Meegahakumbura, M. K., Chalo, R., Kamunya, S., Muchugi, A., Xu, J. C., et al. (2016). Nuclear microsatellites reveal the genetic architecture and breeding history of tea germplasm of East Africa. Tree Genet. Genomes 12, 11. doi: 10.1007/s11295-015-0963-x

Wight, W. (1959). Nomenclature and classification of the tea plant. Nature 183, 1726-1728. doi: 10.1038/1831726a0

Wight, W. (1962). Tea classification revised. Curr. Sci. 31, 298-299.

Wolfe, K. H., Li, W. H., and Sharp, P. M. (1987). Rates of nucleotide substitution vary greatly among plant mitochondrial, chloroplast, and nuclear DNAs. Proc. Natl. Acad. Sci. U.S.A. 84, 9054-9058. doi: 10.1073/pnas.84.24.9054

Xie, W., Wang, G., Yuan, M., Yao, W., Lyu, K., Zhao, H., et al. (2015). Breeding signatures of rice improvement revealed by agenomic variation map from a large germplasm collection. Proc. Natl. Acad. Sci. U.S.A. 112, 5411-5419. doi: $10.1073 /$ pnas. 1515919112

Yang, C. S., Wang, X., Lu, G., and Picinich, S. C. (2009). Cancer prevention by tea: animal studies, molecular mechanisms and human relevance. Nat. Rev. Cancer 9, 429-439. doi: 10.1038/nrc2641

Yang, J. B., Yang, S. X., Li, H. T., Yang, J., and Li, D. Z. (2013). Comparative chloroplast genomes of Camellia Species. PLOS ONE 8:e73053. doi: 10.1371/journal.pone.0073053

Yao, M. Z., Chen, L., and Liang, Y. R. (2008). Genetic diversity among tea cultivars from China, Japan and Kenya revealed by ISSR markers and its implication for parental selection in tea breeding programs. Plant Breed. 127, 166-172. doi: 10.1111/j.1439-0523.2007.01448.x

Yao, M. Z., Ma, C. L., Qiao, T. T., Jin, J. Q., and Chen, L. (2012). Diversity distribution and population structure of tea germplasms in China revealed by EST-SSR markers. Tree Genet. Genomes 8, 205-220. doi: 10.1007/s11295-0110433-Z

Yin, H. I., Deng, Y. F., Wang, H. F., Liu, W. G., Zhuang, X. Y., and Chu, W. H. (2015). Tea polyphenols as an anti-virulent compound disrupt quorum sensing regulated pathogenicity of Pseudomonas aeruginosa. Sci. Rep. 5:16158. doi: $10.1038 /$ srep 16158

Conflict of Interest Statement: The authors declare that the research was conducted in the absence of any commercial or financial relationships that could be construed as a potential conflict of interest.

The reviewer LB and handling Editor declared their shared affiliation, and the handling Editor states that the process nevertheless met the standards of a fair and objective review.

Copyright (C) 2016 Wambulwa, Meegahakumbura, Kamunya, Muchugi, Möller, Liu, $X u$, Ranjitkar, Li and Gao. This is an open-access article distributed under the terms of the Creative Commons Attribution License (CC BY). The use, distribution or reproduction in other forums is permitted, provided the original author(s) or licensor are credited and that the original publication in this journal is cited, in accordance with accepted academic practice. No use, distribution or reproduction is permitted which does not comply with these terms. 